\title{
Memory for recent actions in the bottlenosed dolphin (Tursiops truncatus): Repetition of arbitrary behaviors using an abstract rule
}

\author{
EDUARDO MERCADO III, SCOTT O. MURRAY, \\ ROBERT K. UYEYAMA, ADAM A. PACK, and LOUIS M. HERMAN \\ Kewalo Basin Marine Mammal Laboratory, University of Hawaii, Honolulu, Hawaii
}

\begin{abstract}
Little is known about how animals represent their own actions in working memory. We investigated whether bottlenosed dolphins could recall actions they had recently performed and reveal those recollections using an abstract rule. Two dolphins were trained to respond to a specific gestural command by repeating the last behavior performed. Both dolphins proved to be able to repeat a wide variety of behaviors on command and were able to generalize the repeating rule to novel behaviors and situations. One dolphin was able to repeat all 36 behaviors she was tested on, including behaviors involving multiple simultaneous actions and self-selected behaviors. These results suggest that dolphins can flexibly access memories of their recent actions and that these memories are of sufficient detail to allow for reenactments. The repeating task can potentially be used to investigate short-term action and event representations in a variety of species.
\end{abstract}

Animals act under the influence of mental representations that result from a variety of internal and external factors. Understanding how animals create, activate, and manipulate such representations is a central concern for studies of animal learning and memory. A variety of techniques (e.g., delayed matching-to-sample tasks and maze tasks) have been developed to gain access to "unobservable" neural representational systems (for a review, see Roitblat, 1982, 1987). These techniques have been used extensively to investigate how animals represent external stimuli (e.g., objects, sounds, locations, and events) that they have experienced in the recent past. In contrast, few studies have investigated how animals represent recent internal events, such as the production of behaviors. Consequently, more is currently known about how animals represent environmental conditions in working memory than about how they represent their recent actions. An animal's short-term memory for its own actions can be interpreted as a type of metaknowledge; self-reports based on such memories can be used to measure an animal's ability to explicitly recall past behaviors (Shimp, 1982).

Portions of this research were supported by a grant from Earthwatch to L. Herman and A. Pack. Many thanks to the people who helped us collect our data, including Amy Cutting, Erin Williams, Carrie Southgate, Toni Cimino, Christa Weiss, Kitty Hudson, Joyce Cranmer, Becca Cowan, Deirdre Killebrew, and numerous other volunteers working at the Kewalo Basin Marine Mammal Laboratory. We also thank H. Roitblat and two anonymous reviewers for their comments on previous versions of the manuscript. Correspondence concerning this article should be addressed to E. Mercado, Kewalo Basin Marine Mammal Laboratory, 1129 Ala Moana Boulevard, Honolulu, HI 96814 (e-mail: mercado (a)hawaii.edu).

-Accepted by previous editor, Robert A. Rescorla
The abilities of animals to demonstrate knowledge of their own actions have been investigated primarily within the context of operant conditioning experiments (see, e.g., Beninger, Kendall, \& Vanderwolf, 1974; Kramer, 1982; Morgan \& Nicholas, 1979; Shimp, 1982, 1983, 1984). For example, Beninger et al. trained rats to press different levers, depending on whether they were engaged in face washing, walking, rearing, or simply remaining still. During training, the rats' behaviors were monitored, and, whenever a rat performed one of the four target behaviors, a buzzer sounded, indicating the activation of four levers. Each lever was associated with a specific behavior; the lever associated with the behavior just performed by the rat was indicated by a cue light. Reinforcement was delivered if the first lever pressed by a rat after the buzzer was the one associated with the behavior just performed (i.e., the lever beneath the cue light). After these associations were trained, the rats were tested on their ability to perform this task without the cue lights. They proved to be capable of performing the task at above-chance levels without light cues, which demonstrated their ability to discriminate the four behavioral states and to associate each with a specific response. These findings with rats were replicated in the study by Morgan and Nicholas.

Shimp $(1981,1982,1983,1984)$ used several different strategies to investigate the extent to which pigeons and rats could report on their own behaviors. Pigeons were trained (1) in symbolic matching-to-sample tasks, in which correct responses were dependent on what patterns of behavior had been performed previously (e.g., patterns of key pecking; Shimp, 1981, 1982, 1983), and (2) with an autoshaping technique, in which reinforcers were automatically delivered after a red or a green keypress, if the preceding interresponse times had been short or long, re- 
spectively (Shimp, 1982). The pigeons were able to discriminate their recent patterns of key pecking at abovechance levels for retention intervals of up to $8 \mathrm{sec}$ (Shimp, 1981). Later tests of rats with an analogous self-report task revealed performance characteristics similar to that of the pigeons (Shimp, 1984). Evidence has also been reported that suggested that a chimpanzee was able to symbolically describe its own behavior (Premack, 1976, 1986). After only minimal training, a chimpanzee produced 54 simple play behaviors; following 11 of these behaviors, the chimpanzee spontaneously selected symbols that represented the most recently performed play act. In no case was a play behavior followed by the selection of a symbol that was unrelated to that behavior.

Other studies may have indirectly demonstrated animals' motor representation capabilities. For example, pigeons' visual matching abilities improve when they are trained to perform sample-specific responses (Urcuioli, 1984; Urcuioli \& DeMarse, 1994; Urcuioli \& Honig, 1980). In some cases, cues associated with differential responding can even overshadow the original sample. One interpretation of these results is that the pigeons were able to use memories of their recent actions to help them choose between alternatives.

Collectively, these studies suggest that (1) several species can encode behaviors they have recently performed, (2) these species can retain representations of recent past behaviors over periods of several seconds or more, and (3) these short-term representations are available to learning and decision-making processes. However, the types of behaviors that animals learned to report on in these experiments were severely constrained, and extensive training was often required to teach the animals a single, highly specific reporting task. In addition, it is possible that, in some cases, discriminations were based on physiological aftereffects associated with specific behaviors or on memories for durations rather than on memories for actions. Consequently, questions about the general capabilities of animals to encode and recall their own behaviors and about the extent and limitations of such abilities are not adequately addressed by these investigations.

Bottlenosed dolphins have demonstrated the ability to learn and generalize a variety of reporting tasks. In particular, they have shown the ability to report on the presence or absence of named objects in their environment (Herman \& Forestell, 1985) and to report, through behavioral mimicry, on the behaviors of other animals, including dolphins, seals, and humans (Herman, Pack, \& Morrel-Samuels, 1993; Tayler \& Saayman, 1973; Xitco, 1988). Mimicry abilities were placed under stimulus control and shown to be generalizable to novel behaviors that had not been previously mimicked or performed and that were not part of the dolphins' natural behavioral repertoire. Tests of delayed behavioral mimicry revealed that the dolphins could correctly mimic behaviors at above-chance levels after delays of as long as $80 \mathrm{sec}$ (Xitco, 1988). The dolphins remained able to correctly mimic behaviors, even when they were required to perform a distractor task during the retention interval, which suggests that the dolphins were internally encoding to-be-mimicked behaviors rather than simply using postural cues to remember behaviors.

Dolphins have also been shown to be capable of reporting on their own behaviors (Taylor, 1995) in a task similar to that used by Beninger et al. (1974). A dolphin was first trained to perform one of five behaviors, depending on which one of five physical symbols it was shown. Next, the dolphin was trained to respond to a single gestural signal by self-selecting and performing any one of the five specific behaviors. The only constraint was that a given action could not be offered more than twice in a row. In the final training stage, the dolphin was presented with the gestural signal. It self-selected and performed one of the five behaviors and then touched one of two physical symbols presented after the self-selected behavior was completed. Reinforcement was given if the dolphin touched the symbol associated with the behavior it had just performed. The dolphins learned to perform this task at above-chance levels, which showed that they could discriminate the five behavioral states and associate those states with particular physical stimuli. In addition, one dolphin showed the ability to self-select behaviors from a set of five, while avoiding repeating subsequent behaviors, at above-chance levels, which suggests that the dolphin was monitoring its last response to the gestural command. Other evidence suggesting that dolphins can monitor their past actions is provided by the ability of dolphins to learn to innovate behaviors upon command, with the constraint that they not repeat an immediately preceding behavior (Braslau-Schneck, 1994; also see Pryor, Haag, \& O'Reilly, 1969). These innovative capabilities suggest that dolphins might be able to selectively perform behaviors that they have not performed previously by monitoring behaviors they have performed in the past.

In the present study, we investigated whether bottlenosed dolphins could recall their own recent behaviors and reveal those recollections on the basis of an abstract (i.e., highly generalizable) rule. Two dolphins were trained to respond to a specific gestural command by repeating the last behavior they performed. Tests of repeating performance were conducted in four parts. In Part A, the dolphins were pretested on their ability to perform the repeating task within formal test trials. In Part $B$, we investigated the range of familiar behaviors the dolphins could successfully repeat on command. In Part C, we tested whether the dolphins could generalize the repeating rule to novel behaviors that they had not previously been instructed to repeat. Finally, in Part D, we tested one dolphin's ability to repeat a behavior multiple times on command.

Unlike previous studies with other species, the behaviors the dolphins were asked to recall in this experiment included a substantial number of the dolphins' trained repertoire of behaviors, as well as combinations of actions not explicitly trained and behaviors self-selected by the dolphins. Additionally, past methodologies have only al- 
lowed researchers to investigate whether animals could discriminate recently performed behaviors and perform arbitrary responses on the basis of those discriminations. In contrast, the methods in the current study require that the dolphins not only discriminate but also identify past actions and perform responses that clearly reveal the extent to which past actions were identified. Finally, because the dolphins were trained to report on their behaviors using an abstract rule, it was possible to test them in a variety of novel situations without any additional training. The results of the current study can potentially address more general questions about (1) the abilities of animals to represent their own recently performed behaviors in working memory, (2) the general accessibility of these representations, and (3) the ability of such representations to affect future behaviors.

\section{PART A}

Pretests were conducted in order to verify that the dolphins had a basic understanding of the repeating task and could perform the task within the constraints of the formal testing procedure. In addition, these pretests were used to identify a set of behaviors that the dolphins were highly proficient at repeating, to serve as a baseline for comparison in later test trials.

\section{Method}

Subjects. The subjects in this study were two 12-year-old Atlantic bottlenosed dolphins, Tursiops truncatus-Hiapo, a male, and Elele, a female. The dolphins were housed together in a sea-water tank 15.2 $\mathrm{m}$ in diameter and $2 \mathrm{~m}$ deep. Each dolphin was fed approximately one quarter of its daily ration of $9.1 \mathrm{~kg}$ of smelt during each experimental session. Both dolphins had received extensive training and had been subjects in a variety of cognitive studies (see, e.g., Herman et al., 1993; Herman, Pack, \& Wood, 1994, Pack \& Herman, 1995).

Procedure. The gestural commands used to instruct the dolphins to perform a particular behavior were moderate- to large-scale movements of the arms and hands of a trainer who stood immediately adjacent to the tank wall; only the upper half of the trainer's body was visible to the dolphin in the tank (for further details, see Herman, Richards, \& Wolz, 1984). Dolphins have been shown to be highly proficient at recognizing such gestures, even when the gestures are substantially degraded (Herman, Morrel-Samuels, \& Pack, 1990). A single gestural command could be used to instruct a dolphin to perform a single action, or multiple consecutive gestural commands could be used to instruct a dolphin to perform several actions simultaneously, such as spitting while jumping (glossed as a combination behavior). The repeat command consisted of a single gesture. Unlike other gestural commands, the repeat command was not associated with a specific action or actions but rather was associated with an abstract rule. Specifically, the repeat command instructed the dolphin to repeat the behavior just performed.

Training for the repeat command was conducted in informal sessions, without specific experimental applications in mind. Consequently, detailed records of early training procedures are unavailable. Generally, a training sequence consisted sequentially of (1) a gestural command that denoted a behavior to be performed, (2) performance of the behavior by the subject, (3) the repeat command, and (4) performance of a second behavior by the subject. The dolphin was rewarded if the behavior that was offered in response to the repeat command was a repetition of the initial behavior performed. Intermittently, the dolphin was cued as to the correct response to a repeat command by immediately following the repeat command with a second gestural command that specifically denoted the correct response. The repeating task was initially trained with a limited set of behaviors, and the set increased with dolphin proficiency.

The dolphins were pretested on their ability to repeat several behaviors that were believed to be relatively simple for them to repeat. Elele was tested in five sessions, whereas Hiapo was tested in six sessions. Sessions consisted of 24 trials each. At the beginning of each trial, the dolphin was given a pay attention gesture by the trainer that instructed it to raise its head out of the water, with its ventral side facing the trainer. A tankside assistant, hidden from the dolphin's view, then verbally informed the trainer of the first gestural command, according to the dictates of a written list. An experimenter (remotely located in an enclosed raised platform overlooking the tank) signaled over a loudspeaker for the trainer to give the gestural command corresponding to the first behavior (B1). The dolphin's performance of $B 1$ was evaluated by the experimenter as either correct or incorrect, and this evaluation was verbally signaled to the trainer. If $B 1$ was performed incorrectly, the trainer signaled the dolphin to return to the station by splashing his or her hand in the water, thus ending the trial. If $B 1$ was performed correctly, the trainer blew a whistle to terminate $\mathrm{Bl}$ and to signal the dolphin to return to the training station; the tankside assistant informed the trainer of a second gestural command, to be given to the dolphin as soon as the trainer blew the whistle. When the dolphin returned to the trainer, the experimenter signaled the trainer verbally to give the gestural command corresponding to a second behavior (B2). The second gestural command instructed the dolphin either to repeat the previous behavior (glossed as repeat trials) or to perform a specific behavior other than the initial behavior (glossed as nonrepeat trials). The dolphin's response to the second gesture was described by a blind observer (located near the experimenter), using prescribed labels corresponding to specific trained behaviors. The blind observer had no knowledge of the gestural command given for $\mathrm{B} 1$ or $\mathrm{B} 2$. If the labeled response indicated that the dolphin had correctly performed B2, the trainer was notified, and he or she blew a whistle. The dolphin then returned to the trainer and was rewarded with social reinforcement (such as vocal praise) and a fish. Otherwise, the trainer simply signaled the dolphin to return to the station by splashing the water.

The ratio of repeat trials to nonrepeat trials varied across sessions. When behaviors within a session involved objects, the objects were placed in the tank before any trials were begun; otherwise, no objects were present in the tank. The objects included toys familiar to the dolphins, such as balls, pipes, baskets, and surfboards. During trials, one or two objects were allowed to float freely in the tank. The objects were repositioned by a tankside assistant during the intervals between trials in order to ensure that the objects were less than $8 \mathrm{~m}$ (the approximate radius of the tank) away from the dolphin when a trial began.

Several controls guarded against inadvertent cuing of the dolphins. The trainer wore opaque goggles to prevent eye gaze cues as well as visual observation of the dolphin's performance. Because the trainer had no knowledge of what the second gestural command would be when the dolphin was executing $\mathrm{B} 1$, he or she could not cue the dolphin as to whether B1 would or would not be repeated. In addition, because the trainer could not monitor the dolphin's responses during a trial, there was no opportunity for the trainer to provide any cues to the dolphin regarding the correctness of its performance. The experimenter and blind observer judging the dolphin's behaviors were located in an enclosed raised platform, out of sight of the dolphin, and therefore could not provide any cues to guide the dolphin's behaviors. 
Table 1

Descriptions of Behaviors and Performance Levels for Each Behavior

Behavior Test Sets Elele Hiapo

Set 1

Make a backward full turn underwater Swim in a circle, belly up, fins waving Leap out of water and spiral

Blow air bubbles underwater

$4 / 4^{*}$

$4 / 4^{*}$

$4 / 4^{*}$

$4 / 4^{*}$

$4 / 4^{*}$

$0 / 3$

$4 / 4^{*}$

Set 2

Swim in a circle with head up Make a sideways full turn underwater Swim in a circle at surface, belly up Assume vertical position, tail up Set 3

Leap over an object

Swim belly up, under an object

Open mouth around an object

Swim with object held between fins

Set 4

Move rostrum up and down

Move rostrum side to side

Move tail side to side

Move tail up and down

$\begin{array}{ll}4 / 4^{*} & 3 / 3^{*} \\ 1 / 4 & 4 / 4^{*} \\ 2 / 4 & 4 / 4^{*} \\ 2 / 4 & 0 / 4\end{array}$

$2 / 4 \quad 0 / 3$

$4 / 4^{*} \quad 0 / 3$

$4 / 4^{*} \quad 2 / 4$

$3 / 3^{*} \quad 3 / 4^{*}$

$4 / 4^{*} \quad 1 / 4$

$4 / 4^{*} \quad 4 / 4^{*}$

$4 / 4^{*} \quad 4 / 4^{*}$

$3 / 4^{*} \quad 4 / 4^{*}$

Set 5

Toss an object with rostrum

Touch an object with fin

Toss an object with tail

Touch an object with tail

$\begin{array}{ll}4 / 4^{*} & 2 / 4 \\ 4 / 4^{*} & 4 / 4^{*} \\ 4 / 4^{*} & 1 / 3 \\ 4 / 4^{*} & 1 / 4\end{array}$

Set 6

Swim in a circle, side up

Swim in a circle, rostrum up, fins waving

Swim in a circle, shaking rostrum

Leap belly up, mouth open

Set 7

Swim in a circle with belly up

Swim in a circle with mouth open

Swim in a circle with tail slapping

Leap belly-up, fins waving

Set 8

Innovate a behavior

Swim in a circle, mouth open, spinning

Swim in a circle, belly up, tail slapping

Leap belly up, mouth open, fins waving

Note-Data are given as number correct divided by number of trials. ${ }^{*} p<.05$, summed binomial test.

\section{Results and Discussion}

Elele showed much better performance levels than Hiapo in initial pretest trials; she correctly repeated behaviors in $104(87 \%)$ out of 120 trials, as compared with Hiapo, who correctly repeated behaviors in $86(62 \%)$ of 138 trials. Both dolphins showed a tendency to be distracted by objects placed in the tank and to perform predictable behaviors when incorrectly repeating a trial. Because Hiapo's performance levels were so much lower than Elele's in early pretest sessions, he was given an extra session in which half of the trials were cued (as previously described). This technique appeared to increase Hiapo's performance levels on cued as well as on noncued trials. Four baseline behaviors were identified, from these initial pretests, that the dolphins were highly proficient at repeating: squirting water out of the mouth (spit), somersaulting vertically underwater (somersault), swimming at the surface with head hunched over (hunch), and leaping out of the water, reentering head first (jump). When Hiapo was tested with only these four behaviors, he showed high performance levels, correctly repeating B1 in $30(94 \%)$ of 32 trials. Elele correctly repeated the four behaviors in $29(100 \%)$ out of 29 trials.

\section{PART B}

The dolphins were formally tested on their ability to repeat 32 behaviors from their trained repertoire. Test behaviors were selected such that the types of actions involved were as diverse as possible and of increasing complexity. The dolphins' ability to repeat self-selected behaviors was also investigated.

\section{Method}

Behaviors were grouped into eight test sets of increasing complexity (see Table 1). Each test set was composed of the four baseline behaviors and four behaviors that had not previously been formally tested. The complexity of a behavior was operationally defined as the number of gestural commands (1-4) that were required to instruct the dolphin to perform the behavior and the number of simultaneous actions that were requested of the dolphin (1-3). Sets 1-5 consisted of behaviors requested with a sequence of one or two gestures, some of which required the dolphin to perform an action to an object. Sets 6 and 7 included combination behaviors requested with two gestural commands. Set 8 consisted of combination behaviors requested with 3-4 gestural commands and one test behavior (requested with a single gesture) that required the dolphin to self-select a behavior. Both dolphins had been previously trained to self-select behaviors in response to a gestural command (glossed as creative). The dolphins could perform any behavior in response to the creative command, except for behaviors that they had recently offered in response to the command. In a previous study, it was found that Elele performed a range of 61 different behaviors in response to the creative command, whereas Hiapo performed a range of 88 different behaviors (Braslau-Schneck, 1994).

The general procedures of Part A were followed. A test set was completed within two sessions of 32 trials each; each session consisted of 8 trials requiring the dolphin to repeat baseline behaviors, 8 trials requiring the dolphin to repeat previously untested behaviors, and 16 nonrepeat trials. Trial types were randomly ordered within a session, and behaviors were randomly ordered within trials; random sequences were generated with computer software. The dolphin had two opportunities within each session to repeat each of the four previously untested behaviors. Because trials in which the dolphin did not correctly perform the first behavior were aborted, it was possible that a dolphin could lose an opportunity to repeat a behavior. Such trials were not rerun, to ensure that both dolphins had equivalent exposure to initial gestural commands within the formal testing context. A session was divided into two blocks of 16 trials; each block of trials was separated by a minimum 2 -min interval. Intertrial intervals and intergesture intervals (i.e., the interval between the gestural command for Bl and the gestural command for B2) were timed with a stopwatch; there was a minimum 30-sec interval between trials.

\section{Results and Discussion}

The dolphins' performance levels on each test behavior are reported in Table 1. Chance performance was con- 
sidered to be one correct repeat in eight trials $(p=.125)$, because there were eight possible correct responses to the repeat command within a session. This is a conservative criterion, because the dolphins could (and did) respond to the repeat command with behaviors other than the eight behaviors they were being tested on within a session. It was assumed that, if the dolphins were guessing responses, their guesses on each trial would be independent of one another. Given that the dolphins were tested only four times at repeating each previously untested behavior, they had to correctly repeat a behavior in at least three out of four trials for their performance to be statistically significant, using the summed binomial test $(p<$ .05 ; assuming independence of trials).

Elele's performance levels overall were high. She correctly repeated test behaviors in $114(90 \%)$ out of 127 trials. Her ability to repeat test behaviors was comparable to her ability to repeat baseline behaviors $(94 \%$ correct). Elele repeated $28(88 \%)$ of the 32 test behaviors at above-chance levels; she was $100 \%$ correct on $24(86 \%)$ of these 28 behaviors. Elele correctly repeated every behavior she was tested on at least once. When Elele made errors in repeating a behavior, she typically performed either (1) an incomplete repetition of the first behaviorfor example, repeating some but not all of the actions $(4 / 13,31 \%) ;(2)$ one of the four baseline behaviors $(5 / 13$, $39 \%)$; (3) one of the other three test behaviors $(2 / 13$, $15 \%)$; or (4) a behavior not included within the test set $(2 / 13,15 \%)$. Elele performed $248(98 \%)$ out of 252 nonrepeat trials correctly; only one of the four errors was an unrequested repetition of the first behavior, indicating that the repeating rule was under stimulus control.

Hiapo did not perform as well as Elele. He correctly repeated test behaviors in $62(57 \%)$ out of 109 trials (the reduced number of repeat trials for Hiapo was a result of his poorer performance on initial behaviors). Hiapo was much better at repeating baseline behaviors, performing 111 $(88 \%)$ out of 126 trials correctly. He performed at abovechance levels on $14(44 \%)$ of the 32 test behaviors; he was $100 \%$ correct on $11(79 \%)$ of these 14 behaviors. Hiapo correctly repeated $22(69 \%)$ of the 32 test behaviors at least once. Like Elele, Hiapo's errors consisted of incomplete repetitions of first behaviors $(4 / 47,9 \%)$, baseline behaviors $(12 / 47,25.5 \%)$, other test behaviors $(12 / 47,25.5 \%)$, or behaviors not included within the test set $(19 / 47,40 \%)$. Hiapo performed $219(90 \%)$ out of 242 nonrepeat trials correctly; only $2(9 \%)$ of the 23 errors were unrequested repetitions of the first behavior.

Elele correctly repeated three out of four self-selected creative behaviors, and Hiapo correctly repeated one out of four self-selected behaviors. Both Elele and Hiapo performed three different behaviors in response to the creative command. The creative command is not associated with a specific behavior. Consequently, the dolphins' ability to repeat actions performed in response to the creative command strongly suggests that they are not simply recalling previous gestures.
There was no evidence that the performance of either dolphin was affected by such factors as complexity (either behavioral or gestural) or the type of behavior performed. However, interpretations of the effects of complexity are confounded, because the dolphins were tested on increasingly complex sets of behaviors, and it is possible that the dolphins' capabilities improved as testing progressed.

The results clearly establish that both dolphins could repeat a wide variety of behaviors on command, including combination behaviors and self-selected behaviors. In addition, it is likely that, during these test sessions, the dolphins repeated many behaviors that they had never been asked to repeat before. However, because initial training sessions were not recorded in any detail, it is impossible to say with certainty which of the 32 behaviors the dolphins had previous experience repeating in informal sessions. Thus, although these results provide some evidence that the dolphins learned a generalized repeating rule, they do not compel this inference.

\section{PART C}

To clearly establish whether the dolphins had learned a generalized repeating rule, the dolphins were trained to perform four novel behaviors and then were tested on their ability to repeat these behaviors on first exposure.

\section{Method}

The four novel behaviors trained for use in this part of the experiment were (1) spinning with head and pectoral fins above the surface of the water (pirouette), (2) submerging beneath the surface (sink), (3) repeatedly throwing a ball in the air and catching it with the mouth (play ball), and (4) pushing a PVC paddle (paddle). Although the dolphins had previous experience in performing behaviors similar to these, they had not been trained to perform stereotyped versions of these behaviors in response to specific gestural commands. Because these four behaviors were trained for use in this experiment, it is certain that the dolphins had no previous experience repeating them in response to the repeat command.

The testing conditions and the general procedures employed were the same as those in Part B. During all trials, a PVC paddle was present on the tank wall (approximately $3 \mathrm{~m}$ from the dolphin's stationing position), and a ball floated freely within the tank.

\section{Results and Discussion}

Table 2 summarizes the dolphins' performance on tests of repetitions of novel behaviors. Both dolphins repeated each of the four novel behaviors correctly at least once out of their four opportunities. Elele correctly repeated novel behaviors in $11(79 \%)$ of 14 trials, whereas Hiapo correctly repeated novel behaviors in $8(50 \%)$ of 16 trials. Elele performed at above-chance levels on two of the four novel behaviors, whereas Hiapo performed at above-chance levels on one of the four behaviors. During tests of novel behaviors, Elele performed $30(97 \%)$ out of 31 nonrepeat trials correctly, and Hiapo performed 30 $(100 \%)$ out of 30 nonrepeat trials correctly; the one error Elele made did not involve an unrequested repetition of the first behavior. 
Table 2

Performance Levels for Repetitions of Novel Behaviors

\begin{tabular}{llc}
\hline Behavior & Elele & Hiapo \\
\hline Pirouette & $4 / 4^{*}$ & $2 / 4$ \\
Sink & $3 / 3^{*}$ & $1 / 4$ \\
Play ball & $2 / 3$ & $4 / 4^{*}$ \\
Paddle & $2 / 4$ & $1 / 4$ \\
\hline
\end{tabular}

Note-Data are given as number correct divided by number of trials. ${ }^{*} p<.05$, summed binomial test.

Anecdotally, the responses that both Hiapo and Elele made in novel test trials may provide some insight into their strategies and repeating capabilities. For example, Elele seemed gradually to learn to recall the paddle behavior. The first time she was instructed to repeat this behavior, she balked (i.e., refused to respond). On her second opportunity, she repeated the behavior correctly, after hesitating approximately $8 \mathrm{sec}$. On her third opportunity, Elele performed an incorrect response (spit); however, when she was signaled to return to the trainer, she instead swam over to the paddle and performed the paddle behavior several times in succession before finally returning. The next time Elele was instructed to repeat the paddle behavior, she did so correctly, without hesitation.

Both Hiapo and Elele developed a similar encoding strategy for performing play ball. Normally (in training sessions), the dolphins would drop the ball and return to the station after completing the behavior. In the context of the experiment, however, they quickly learned to keep the ball in their mouths, anticipating the possibility of a repeat command. Because no constraints were placed on how the dolphins could encode behaviors, this strategy was ignored for purposes of evaluating the dolphin's performance of the second behavior.

Overall, these results provide compelling evidence that both dolphins learned a generalized repeating rule. Unlike two-alternative forced-choice tasks that potentially allow subjects to learn correct responses quickly using rules of exclusion and association (see, e.g., Schusterman, Gisiner, Grimm, \& Hanggi, 1993), an incorrect response to the repeat command provides little information regarding what the correct response should have been. Consequently, performance in the first four exposures provides a stringent test of immediacy of transfer.

\section{PART D}

The dolphins' ability to repeat self-selected behaviors provides some evidence that they were recalling past behaviors rather than gestural commands. To further verify that the dolphins were recalling their past actions, Elele was tested on her ability to repeat behaviors multiple times on command. If the rule she had learned to follow when given a repeat command was repeat the behavior corresponding to the previous gesture, it would be difficult for her to respond correctly when the previous gesture was another repeat command (because this command is not associated with a specific behavior). However, if the rule she had learned was repeat the previous action(s), one would expect multiple repeat commands to be no more difficult than single repeat commands.

\section{Method}

The testing conditions and the general procedures employed were the same as those in Parts B and C. Two sessions were run in which Elele was presented with trials in which she was given two repeat commands in succession within a single trial (glossed as double-repeat). For example, after Bl was completed, Elele was instructed to repeat the previous behavior (B1); when she returned from repeating $\mathrm{B} 1$, she was again instructed to repeat the previous behavior. Correct performance thus required that she repeat $B 1$ twice within a single trial. These test trials were conducted with only the four baseline behaviors. A total of 16 test trials were conducted (4 with each baseline behavior). Elele had never been exposed to multiple consecutive repeat commands prior to these test trials.

\section{Results and Discussion}

Elele performed $16(100 \%)$ of the 16 double-repeat trials correctly. She showed no hesitation in performing behaviors a third time when she was presented with two successive repeat commands. Her ceiling level performance indicates that she was recalling actions rather than gestures. In addition, her ability to immediately adapt to these novel trial types further demonstrates the flexibility with which she could apply the repeating rule.

\section{GENERAL DISCUSSION}

Both Hiapo and Elele demonstrated the ability to perform the repeating task with a variety of behaviors of varying complexity. Elele successfully repeated every behavior she was tested on at least once and repeated most behaviors without error ( 26 behaviors total out of 36 tested, $72 \%$ ). In addition, both dolphins demonstrated that they had learned a generalized rule of repeating by correctly applying the repeating rule to novel behaviors. Both dolphins correctly repeated all four novel behaviors at least once in their first four exposures; Elele correctly repeated two novel behaviors without error, and Hiapo correctly repeated one novel behavior without error. To our knowledge, this constitutes the first report of such abilities in any nonhuman species.

During testing, the dolphins could have adopted a strategy of randomly performing one of the behaviors within a test set in response to the repeat command (as was assumed in setting the probability of chance success at .125); however, there was little evidence to suggest that such a strategy was used. Hiapo and Elele did sometimes perform baseline behaviors or other behaviors within the test set when incorrectly repeating a behavior ( 31 of 60 errors, $52 \%$ ), which suggests that the dolphins may have "guessed" a behavior that they had correctly repeated in previous trials when they were unsure of what behavior to perform. However, other error responses, such as incom- 
plete repetitions or behaviors that were not part of the test set, were almost as common ( 29 of 60 errors, $48 \%$ ) as responses that were behaviors within the test set.

Although the dolphins were clearly proficient at repeating behaviors, there is some question as to whether this performance provides evidence that the dolphins had knowledge of their past actions. There are several alternative explanations that could potentially explain this ability. For example, because there was no controlled delay between the gestural command for the first behavior and the gestural command for repetition of that behavior, it could be argued that, instead of recalling the first behavior, the dolphins were simply continuing the first behavior until they were rewarded or instructed to perform a different behavior. Although this explanation may account for the dolphins' successful repetition of a subset of the behaviors, it cannot account for their ability to repeat behaviors that required them to discontinue the first behavior and return to the trainer before receiving the repeat command (e.g., jumps, behaviors performed underwater, behaviors performed away from the training station, and behaviors involving objects such as paddles). It seems clear that the dolphins must have retained some information about past events, to be able to repeat such behaviors so successfully.

Given that the dolphins were somehow encoding information about past events, what information was being encoded and how was it being encoded? Although the dolphins could have encoded the gestural commands they received from the trainer and then recalled these commands on viewing the repeat command, several pieces of evidence argue against this possibility. For example, neither dolphin showed a decrement in performance when required to repeat behaviors whose instructions involved as many as four gestural commands. More importantly, Elele's successful performances in trials in which she was given multiple repeat commands and in trials in which she was instructed to repeat a self-selected behavior provides strong evidence that she was recalling past actions rather than past gestural commands.

Perhaps the simplest way for a dolphin to encode its past actions would be through postural coding strategies. In some trials, the dolphins appeared to take advantage of such strategies (the extent of such posturing was not explicitly measured). For example, when the first behavior required that the dolphin perform a behavior with its mouth open, the dolphin might continue to keep its mouth open until the gestural command for the second behavior was given. The dolphins' tendency to keep a ball in their mouth after performing the play ball behavior can also be interpreted as a type of postural coding. Are such postural codes sufficient to explain the dolphins' repeating performances? Certainly they can potentially explain the dolphins' ability to repeat some behaviors; however, it seems unlikely that the dolphins relied exclusively on this strategy. For example, some test behaviors required the dolphins to perform several actions simultaneously, such as performing a jump with belly up, pectoral fins waving and mouth open, or swimming in a circle with belly up and tail slapping. Elele was able to repeat such behaviors without error. If she was able to somehow posturally encode such complex behaviors while swimming back to station, she must have used rather complex postural coding techniques. These techniques would have to be flexible enough that they could be used to encode a wide variety of behaviors (including novel behaviors) and subtle enough that they were not evident to trained observers. Furthermore, to account for Elele's ability to immediately perform correctly from the first trial in response to multiple sequential repeats, one would have to conclude that the dolphins utilize this postural coding system after almost every behavior they perform.

The simplest explanation that can account for all of the results in this study is that the dolphins can retain internal representations of their recent past actions in working memory that they can use to recreate those past actions. These representations must be flexibly accessible and detailed enough to allow for the repetition of fairly complex behaviors. Unlike previous studies (Beninger et al., 1974; Kramer, 1982; Morgan \& Nicholas, 1979; Shimp, 1982, 1983, 1984; Urcuioli, 1984; Urcuioli \& DeMarse, 1994; Urcuioli \& Honig, 1980), which only showed that animals were capable of discriminating their past behaviors, our results clearly demonstrate that dolphins can specifically identify actions that they have recently performed.

Killeen (1994) has suggested that reinforcement acts on events in short-term memory, and that what is reinforced is an animal's representation of a behavior rather than the behavior itself. If this is the case, an animal's ability to learn novel behaviors is limited not only by physical constraints but also by its ability to represent behaviors in working memory. Consequently, one would expect that animals that have demonstrated the ability to flexibly learn complex behaviors could be shown to have correspondingly flexible systems for mentally representing those behaviors. The current study provides evidence suggesting that, at least in the case of the bottlenosed dolphin, such expectations are well founded.

The repeating rule learned by the dolphins could be viewed as a self-imitation concept. Dolphins are one of the few species that have been shown to be capable of learning to imitate on command and the only nonhuman species that has been shown to be capable of imitating both sounds and actions on command (Herman et al., 1993; Richards, 1986; Richards, Wolz, \& Herman, 1984; Xitco, 1988). Only chimpanzees have shown comparable imitation capabilities. For example, chimpanzees have been trained to imitate arbitrary behaviors in response to the vocal command Do this! (Custance, Whiten, \& Bard, 1995; Hayes \& Hayes, 1952; see also Tomasello, Savage-Rumbaugh, \& Kruger, 1994). In comparison, the dolphins' repeat command might be glossed as Do that again!

Imitation has generally been considered to be a unique form of social or observational learning (see, e.g., Galef, 1988; Heyes, 1994; Whiten \& Hamm, 1992). From this 
perspective, the concept of self-imitation seems somewhat wrongheaded. However, imitation can also be viewed as a process whereby externally (e.g., visually) activated motor representations are used to generate actions (Jeannerod, 1994). If this interpretation is correct, the primary difference between imitation of others and imitation of self is the stimuli that activate the motor representation. The recent discovery of mirror neurons in monkeys provides some support for this account (Di Pellegrino, Fadiga, Fogassi, Gallese, \& Rizzolatti, 1992; Gallese, Fadiga, Fogassi, \& Rizzolatti, 1996; Rizzolatti, Fadiga, Gallese, \& Fogassi, 1996). These neurons fire both when a monkey performs an action and when it observes other monkeys or humans perform similar actions. However, the generally unimpressive imitation capabilities of monkeys (see, e.g., Mitchell \& Anderson, 1993) suggest that the ability to imitate requires more than just a system for matching observed and executed actions. Neuropsychological studies that directly compare the neural activity of animals imitating and repeating behaviors may clarify the relationship between these two abilities.

The repeating task provides a highly flexible way of investigating animals' representations of past actions and past events that can potentially be applied to a variety of species. Performance on the task could serve as a nonverbal measure of an animal's explicit memory for implicitly learned skills. Just as delayed matching-to-sample tasks have revealed much about animals' representations of objects and maze learning tasks have provided information about animals' spatial representations, the repeating task can potentially increase our understanding of how animals represent their own actions. In particular, assessments of various species' ability to maintain and recall representations of recent actions can reveal the flexibility and specificity of animals' short-term representations of those actions and can further elucidate the influence that internal and external factors have on animals' actions.

\section{REFERENCES}

Beninger, R. J., Kendall, S. B., \& Vanderwolf, C. H. (1974). The ability of rats to discriminate their own behavior. Canadian Journal of Psychology, 28, 79-91.

BRASLAU-SCHNECK, S. (1994). Innovative behaviors and synchronization in bottlenosed dolphins. Unpublished master's thesis, University of Hawaii, Honolulu.

Custance, D. M., Whiten, A., \& Bard, K. A. (1995). Can young chimpanzees (Pan troglodytes) imitate arbitrary actions? Hayes \& Hayes (1952) revisited. Behaviour, 132, 837-859.

Di Pellegrino, G., Fadiga, L., Fogassi, L., Gallese, V., \& RizzoLATTI, G. (1992). Understanding motor events: A neurophysiological study. Experimental Brain Research, 91, 176-180.

GALEF, B. G., JR. (1988). Imitation in animals: History, definitions and interpretation of data from the psychological laboratory. In T. Zentall \& B. Galef (Eds.), Social learning: Psychological and biological perspectives (pp. 3-28). Hillsdale, NJ: Erlbaum.

Gallese, V., Fadiga, L., Fogassi, L., \& Rizzolatti, G. (1996). Action recognition in the premotor cortex. Brain, 119, 593-609.

HAYES, K. J., \& HAYES, C. (1952). Imitation in a home-raised chimpanzee. Journal of Comparative Physiology \& Psychology, 45, 31 -46.

Herman, L. M., \& Forestell, P. H. (1985). Reporting presence or absence of named objects by a language trained dolphin. Neuroscience \& Biobehavioral Reviews, 9, 667-691.
Herman, L. M., Morrel-Samuels, P., \& Pack, A. A. (1990). Bottlenosed dolphin and human recognition of veridical and degraded displays of an artificial gestural language. Journal of Experimental Psychology: General, 119, 215-230.

Herman, L. M., PaCk, A. A., \& Morrel-Samuels, P. (1993). Representational and conceptual skills of dolphins. In H. R. Roitblat, L. M. Herman, \& P. Nachtigall (Eds.), Language and communication: Comparative perspectives (pp. 299-308). Hillsdale, NJ: Erlbaum.

Herman, L. M., Pack, A. A., \& Wood, A. M. (1994). Bottlenosed dolphins can generalize rules and develop abstract concepts. Marine Mammal Science, 10, 70-80.

Herman, L. M., Richards, D. G., \& Wolz, J. P. (1984). Comprehension of sentences by bottlenosed dolphins. Cognition, 16, 129-219.

HeYes, C. M. (1994). Social learning in animals: Categories and mechanisms. Biological Review, 69, 207-231.

JEANNEROD, M. (1994). The representing brain: Neural correlates of motor intention and imagery. Behavioral \& Brain Sciences, 17, 187-245.

KILLEEN, P. R. (1994). Mathematical principles of reinforcement, Behavioral \& Brain Sciences, 17, 105-172.

KRAMER, S. P. (1982). Memory for recent behavior in the pigeon. Journal of the Experimental Analysis of Behavior, 38, 71-85.

Mitchell, R. W., \& ANDERSON, J. R. (1993). Discrimination learning of scratching, but failure to obtain imitation and self-recognition in a long-tailed macaque. Primates, 34, 301-309.

MoRgan, M. J., \& Nicholas, D. J. (1979). Discrimination between reinforced action patterns in the rat. Learning \& Motivation, 10, 1-22.

Pack, A. A., \& Herman, L. M. (1995). Sensory integration in the bottlenosed dolphin: Immediate recognition of complex shapes across the senses of echolocation and vision. Journal of the Acoustical Society of America, 98, 722-733.

Premack, D. (1976). Intelligence in ape and man. Hillsdale, NJ: Erlbaum.

Premack, D. (1986). Gavagai! Or the future history of the animal language controversy. Cambridge, MA: MIT Press.

Pryor, K., HaAg, R., \& O'ReIlly, J. (1969). The creative porpoise: Training for novel behavior. Journal of the Experimental Analysis of Behavior, 12, 653-661.

RicharDs, D. G. (1986). Dolphin vocal mimicry and vocal object labeling. In R. J. Schusterman, J. A. Thomas, \& F. G. Wood (Eds.), Dolphin cognition and behavior: A comparative approach (pp. 273-288). Hillsdale, NJ: Erlbaum

Richards, D. G., Wolz, J. P., \& Herman, L. M. (1984). Vocal mimicry of computer-generated sounds and vocal labeling of objects by a bottlenosed dolphin, Tursiops truncatus. Journal of Comparative Psychology, 98, 10-28.

Rizzolatti, G., Fadiga, L., Gallese, V., \& Fogassi, L. (1996). Premotor cortex and the recognition of motor actions. Cognitive Brain Research, 3, 131-141

RoITBLAT, H. L. (1982). The meaning of representation in animal memory. Behavioral \& Brain Sciences, 5, 353-406.

Roltblat, H. L. (1987). Introduction to comparative cognition. New York: Freeman.

Schusterman, R. J., Gisiner, R., Grimm, B. K., \& HangGi, B. (1993). Behavior control by exclusion and attempts to establish semanticity in marine mammals using match-to-sample paradigms. In H. R. Roitblat, L. M. Herman, \& P. Nachtigall (Eds.), Language and communication: Comparative perspectives (pp. 249-274). Hillsdale, NJ: Erlbaum.

SHImP, C. P. (1981). The local organization of behavior: Discrimination of and memory for simple behavioral patterns. Journal of the Experimental Analysis of Behavior, 36, 303-315.

SHIMP, C. P. (1982). On metaknowledge in the pigeon: An organism's knowledge about its own behavior. Animal Learning \& Behavior, 10, 358-364.

SHIMP, C. P. (1983). The local organization of behavior: Dissociations between a pigeon's behavior and self-reports of that behavior. Journal of the Experimental Analysis of Behavior, 39, 61-68.

SHIMP, C. P. (1984). Self reports by rats of the temporal patterning of their behavior: A dissociation between tacit knowledge and knowledge. In H. R. Roitblat, T. G. Bever, \& H. S. Terrace (Eds.), Animal cognition (pp. 215-229). Hillsdale, NJ: Erlbaum.

TAYLER, C. K., \& SAAYMAN, G. S. (1973). Imitative behavior of Indian 
Ocean bottlenose dolphins (Tursiops aduncus) in captivity. Behaviour, 44, 286-298.

TAYLOR, K. B. (1995). Self-selection and self-monitoring of behavior by bottlenosed dolphins (Tursiops truncatus). Unpublished master's thesis, University of Hawaii, Honolulu.

Tomasello, M., Savage-Rumbaugh, S., \& Kruger, A. C. (1994). Imitative learning of actions on objects by children, chimpanzees, and enculturated chimpanzees. Child Development, 64, 1688-1705.

URCUIOLI, P. J. (1984). Overshadowing in matching-to-sample: Reduction in sample-stimulus control by differential sample behaviors. $A n$ imal Learning \& Behavior, 12, 256-264.

URCUIOLI, P. J., \& DEMARSE, T. (1994). On the relationship between differential outcomes and differential sample responding in match- ing-to-sample. Journal of Experimental Psychology: Animal Behavior Processes, 20, 249-263.

URCUioli, P. J., \& Honig, W. K. (1980). Control of choice in conditional discriminations by sample-specific behaviors. Journal of $E x$ perimental Psychology: Animal Behavior Processes, 6, 251-277.

WhITEN, A., \& HAMM, R. (1992). On the nature and evolution of imitation in the animal kingdom: Reappraisal of a century of research. Advances in the Study of Behavior, 21, 239-283.

XITCO, M. J., JR. (1988). Mimicry of modeled behaviors by bottlenosed dolphins. Unpublished master's thesis, University of Hawaii, Honolulu.

(Manuscript received June 16, 1997 ; revision accepted for publication February 3, 1998.) 